\title{
Update on the Implementation of NCCN Guidelines for Distress Management by NCCN Member Institutions
}

\author{
Kristine A. Donovan, PhD, MBA; ${ }^{\mathrm{a}}$ Teresa L. Deshields, $\mathrm{PhD}^{\mathrm{b}}$; Cheyenne Corbett, $\mathrm{PhD}^{\mathrm{c}}$; and Michelle B. Riba, MD, MS
}

\begin{abstract}
The first NCCN Clinical Practice Guidelines in Oncology (NCCN Guidelines) for Distress Management were published in 1999. Since then, a number of other organizations have advocated for distress screening. Previous surveys of distress screening showed modest progress in implementation of the NCCN Guidelines for Distress Management by NCCN Member Institutions (Mls); this review examined whether further progress has been made. Representatives appointed to the NCCN Distress Management Panel or their designee were asked to complete an online survey in the summer of 2018. The survey was developed based on similar surveys performed in 2005 and 2012 and a survey of psychosocial staffing conducted in NCCN Mls in 2012. New items solicited details about triaging, rescreening, formal screening protocols, and tracking of distressed patients. The survey was completed by representatives from 23 of $27 \mathrm{NCCN} \mathrm{Mls} \mathrm{(85 \% ).}$ Among the responding institutions, 20 (87\%) currently conduct routine screening for distress and 3 are piloting routine screening. All respondents reported use of a self-report measure to screen for distress, with the Distress Thermometer most often used. A total of $70 \%$ of respondents rescreen patients for distress and $83 \%$ reported having a formal distress screening protocol in place. Once triaged, $65 \%$ of institutions who routinely screen for distress track clinical contacts and referrals; $70 \%$ track rates of adherence to screening protocols. Findings suggest wide acceptance and implementation of the NCCN Guidelines. Most respondents reported the existence of a formal distress screening protocol, with routine tracking of clinical contacts and referrals and rates of protocol adherence. Clinical experience and the American College of Surgeons Commission on Cancer accreditation standard for cancer centers appear to have resulted in greater adoption and implementation of the guidelines, but considerable opportunities for improvement remain.
\end{abstract}

J Natl Compr Canc Netw 2019;17(10):1251-1256 doi: $10.6004 /$ jnccn.2019.7358

a Moffitt Cancer Center, Tampa, Florida; ${ }^{b}$ Washington University School of Medicine, St. Louis, Missouri; 'Duke Cancer Institute, Durham, North Carolina; and ${ }^{d}$ University of Michigan, Ann Arbor, Michigan.
NCCN was among the first voices calling for routine screening of distress in patients with cancer. The first NCCN Clinical Practice Guidelines in Oncology (NCCN Guidelines) for Distress Management were published in 1999. ${ }^{1}$ Since then, a number of other organizations have advocated for screening, including the Institute of Medicine, ${ }^{2}$ ASCO ${ }^{3}$ and, in a joint statement, the American Psychosocial Oncology Society, Association of Oncology Social Work, and Oncology Nursing Society. ${ }^{4}$ The American College of Surgeons Commission on Cancer's (ACS CoC) mandate for implementing a systematic protocol for distress screening and referral made screening a requirement for cancer center accreditation. ${ }^{5}$ The mandate took effect in 2015 , spurring the development of processes for distress screening and referral to psychosocial care in most US cancer centers.

To determine the extent to which NCCN Member Institutions (MIs) were implementing key aspects of the NCCN Guidelines for Distress Management, Jacobsen and Ransom ${ }^{6}$ surveyed panel members in 2005. Their findings, published in 2007, suggested a general acceptance of routine screening for distress as part of clinical care, with $53 \%$ of represented MIs conducting routine screening. In 2012, an expanded survey was distributed to NCCN MIs, soliciting more details about screening procedures, use of self-report measures, and the provision of services to distressed patients. The results, published in 2013, suggested modest progress in implementation of guideline recommendations for distress screening $^{7} ; 70 \%$ of respondents reported conducting routine screening, with $79 \%$ of these institutions also rescreening patients. The publication of the 2016 ACS CoC standards manual brought increased documentation requirements and clarified screening and referral processes. ${ }^{8}$ Plans to monitor compliance with these standards as part of the accreditation process seem likely to have motivated more MIs to develop formal distress screening programs. Thus, we sought to examine whether further progress has been made in implementation of the NCCN Guidelines for Distress Management since the previous survey in 2012. 


\section{Methods}

We developed a survey similar in content to the ones used previously $y^{6,7,9}$ and designed to solicit more details about formal screening protocols, tracking and triage of distressed patients, and rescreening. Survey items focused on how positive screens are documented, how and by whom distressed patients are screened and triaged, protocols for service referrals, and availability of psychosocial resources. After pilot testing, the final version was formatted as an online survey. A letter containing a link to the survey was sent via email in June 2018 to the 27 NCCN MIs, 25 of which had a representative appointed to the NCCN Distress Management Panel (Note: NCCN comprised 27 MIs until November 2018); for those without representation on the panel, an invitation to participate was sent to personnel considered likely to be involved with their institution's distress management program. Multiple reminders were sent via email in June through August 2018 to institutional representatives who did not complete the survey in response to the initial request.

\section{Results}

Responses were received from representatives of 23 of the 27 NCCN MIs, reflecting an $85 \%$ response rate. Initial survey items focused on whether, consistent with the current guidelines, patients are screened routinely for distress. Of the 23 responding institutions, 20 (87\%) reported that they currently conduct routine screening for distress and 3 reported they are piloting routine screening. Among the 23 institutions reportedly already screening, 6 (26\%) endeavor to screen all patients, 13 (57\%) to screen outpatients only, $0(0 \%)$ to screen inpatients only, and $4(17 \%)$ to screen select groups of patients (ie, patients at a certain point in the care trajectory, those with certain types of cancer, and/or those undergoing specific treatments).

Regarding personnel who administer the screening tool and methods used to screen, those most often tasked specifically with screening include nurses, medical assistants, and social workers. Table 1 lists the personnel who screen patients at NCCN MIs. The screen is administered via paper and pencil at 12 institutions (52\%) and electronically at 12 (52\%; 10 on-site and 2 off-site); 7 institutions (30\%) administer the measure via interview, with 6 conducting the interview in person and 1 via telephone. More than one approach is used to administer the screen at 8 institutions (35\%), and thus percentages add up to $>100 \%$.

Additional survey items sought information about the triaging and disposition of patients identified as distressed. Among the 20 institutions conducting routine screening

\begin{tabular}{|lcc|}
\hline $\begin{array}{l}\text { Table 1. Personnel Who Conduct Distress } \\
\text { Screening and Patient Triage }\end{array}$ & \\
\hline Personnel & Screening, $\mathbf{n}$ & Triage, $\mathbf{n}$ \\
\hline Medical assistants & 12 & 8 \\
\hline Social workers & 8 & 6 \\
\hline Mental health therapists (other than social workers) & 1 & 2 \\
\hline Nurses & 13 & 13 \\
\hline Advance practice professionals ${ }^{\mathrm{b}}$ & 2 & 7 \\
\hline Physicians & 2 & 8 \\
\hline Clinical psychologists & 2 & 1 \\
\hline Chaplains & 1 & 1 \\
\hline Lay patient navigators & 1 & 0 \\
\hline
\end{tabular}

aNurse practitioners, physician assistants, and clinical nurse specialists. bPractitioners with terminal master's degrees, such as professional counselors and licensed mental health counselors.

and 1 of the institutions piloting screening ( 2 of the $3 \mathrm{did}$ not report results specific to triage), responses indicated that 7 institutions (33\%) triage patients automatically based on computer-generated results, and personnel at 14 institutions (67\%) review the screening result and generate a referral. Personnel most often responsible for further assessment and referral to appropriate services of distressed patients include nurses, medical assistants, and physicians. Table 1 lists the personnel responsible for patient triage. In addition to documentation of a positive screen, the action taken in response to a positive screen is documented automatically in the electronic record at 6 institutions, via chart entry by clinical staff at 14 institutions, and in a visit-specific clinical note at 9 institutions. Some institutions document the response in more than one way, and therefore these numbers add up to $>21$.

All responding institutions use a self-report measure to screen for distress. A total of 11 institutions (48\%) use the NCCN Distress Thermometer, most often alone. Ten institutions (43\%) use the Patient Health Questionnaire (PHQ-2 or PHQ-9), and 4 (17\%) of these also use the Generalized Anxiety Disorder Assessment (GAD-2 or GAD-7). Of the 10 institutions that use the Patient Health Questionnaire (with or without the GAD), 8 also use additional self-report measures, none of which is used by $>1$ institution. These measures include published tools such as the Columbia-Suicide Severity Rating Scale and institutionspecific measures consisting of several single items. Results of distress screening become part of the patient's medical record at 21 institutions; 2 of the 3 institutions piloting screening did not respond to this question.

The next survey items inquired about whether patients are rescreened for distress and, if so, at what 
intervals and how often. A total of 16 institutions (70\%) rescreen the same patients for distress, although the intervals and frequency varied widely, and included daily for inpatients; weekly; every 30, 60, and 90 days; upon referral to other oncologists; at appropriate triggers in the course of disease and treatment; and, for patients with a positive screen, at all subsequent appointments until below threshold.

Finally, the last screening-related survey item sought information about the top 3 distress screening barriers. Table 2 lists the perceived barriers and the number of endorsing respondents. The top 3 barriers were "time necessary to accomplish screening is too great," "inadequate psychosocial staffing," and "providers are not aware of the process."

The next survey items referred to formal screening protocols, referrals, and tracking of referrals. When asked whether there is a formal distress screening protocol in place that ensures patients screening positive are referred for services, 19 respondents $(83 \%)$ indicated that patients are referred inside their healthcare system, and $4(17 \%)$ responded that there is no formal protocol in place, thus patients who screen positive are not referred for services. The latter 4 institutions account for those currently piloting distress screening and 1 institution currently conducting routine screening. When a patient is triaged, 13 of 20 institutions (65\%) who routinely screen patients track clinical contacts and referrals, 7 (35\%) do not. Similarly, 14 institutions (70\%) reportedly track rates of adherence to distress screening protocols, whereas $6(30 \%)$ do not.

Follow-up survey items sought information about the top 3 barriers and facilitators to referral to psychosocial services. Table 3 presents the perceived barriers and facilitators and the number of respondents endorsing each. The top 3 barriers identified were "inadequate psychosocial care staffing," "providers are not

\section{Table 2. Barriers to Routine Distress Screening}

\begin{tabular}{|lc|}
\hline Barrier & Responses, $\mathbf{~}$ \\
\hline Time necessary to accomplish screening is too great & 13 \\
\hline Inadequate psychosocial care staffing & 12 \\
\hline Providers are not aware of the process & 6 \\
\hline Burden is too great for the institution & 5 \\
\hline Lack of institutional support & 4 \\
\hline Providers are not aware of the need & 4 \\
\hline Providers are not supportive of the process & 3 \\
\hline Information technology challenges & 2 \\
\hline Providers assert that they do this already & 1 \\
\hline Providers believe their patients do not need it & 1 \\
\hline
\end{tabular}

aware of the scope of psychosocial services," and time necessary to access services is too great." The top 3 facilitators were "providers believe services are a part of comprehensive patient care," "providers see the need for these services," and "patients themselves ask for these services."

The next survey items were adapted from a previous survey regarding current staffing for psychosocial services at NCCN MIs. ${ }^{9}$ In the current survey, when asked about service models, 14 respondents $(61 \%)$ reported that psychosocial services were both a stand-alone entity (eg, a psychiatry/psychosocial service is clearly defined as a department or program) and integrated/embedded throughout the institution (eg, social workers or other mental health providers are assigned to radiation oncology or a medical oncology service). In contrast, 6 respondents (26\%) reported a stand-alone service only and $3(13 \%)$ reported integrated/embedded services only. Consistent with the service model endorsed, most respondents reported that their institution's psychosocial providers primarily reported to departments or divisions reflecting their professional identity. For example, psychiatrists and psychologists tended to report to a department of psychiatry or supportive care, whereas social workers tended to report to a social work

\section{Table 3. Barriers and Facilitators to Referral to Psychosocial Services}

\section{Barrier}

Responses, $\mathbf{n}$

Inadequate psychosocial care staffing 15

Providers are not aware of the scope of psychosocial services

Time necessary to access services is too great

Providers are not aware of the services

Providers are not aware of the benefits of psychosocial care

Providers who are not psychosocial clinicians

but insist that they provide adequate

psychosocial care

Patient-specific factors

Financial cost to patients is too high

Late referrals (patient's disease is too advanced

to benefit from referral

Providers insist their patients do not need

psychosocial care

Facilitator

Providers believe services are a part of

comprehensive patient care

Providers see the need for these services

Patients themselves ask for these services

A positive distress screen

Providers believe services increase patient

satisfaction 
department positioned within the institution's administrative structure.

Table 4 presents the range of psychosocial staff employed at MIs. In addition to providing common psychosocial services, such as medication management, psychotherapy, financial/transportation/housing needs, and spiritual care, some sites offered relatively unique programs, such as cognitive rehabilitation, medical family therapy, child life services, and recreation therapy. Staff identified in the "other" category included a patient resource coordinator, cancer information resource nurse, art and music therapists, medical family therapists, and trainees. With regard to funding of psychosocial services and staff positions (see Table 4), most are supported by institutional funds. This is particularly true for social workers, nurses, chaplains, psychosocial researchers, and administrative staff. Psychologists and psychiatrists were also supported by institutional funds and were the personnel most likely to be funded by fee-for-service income. Of note, only two-thirds of the MIs who responded to the survey provided complete data related to psychosocial staffing and funding.

A total of 17 institutions (74\%) reported documenting, for tracking purposes, the types of psychosocial services provided, whereas $6(26 \%)$ do not. Additionally, a number of institutions' psychosocial services collect the following data: 11 on patient and/or family satisfaction, 1 on referrer satisfaction, 16 on program utilization or number of referrals, 6 on patient clinical outcomes, and 4 on patient utilization outcomes.
Finally, 9 respondents $(39 \%)$ reported that their institutions provide funding for the psychosocial service and/or providers to conduct research.

\section{Discussion}

Results of the current survey suggest that routine screening for distress among patients with cancer has been widely implemented among NCCN MIs, with essentially all who responded currently conducting or piloting routine screening. Since the ACS CoC made systematic screening an accreditation standard for cancer centers in 2015, this result was expected. Use of many of the same questions from previous surveys allows us to expand on this finding and describe some changes over time in screening practices. The percentage of institutions reporting the screening of all patients has decreased to $26 \%$ from $30 \%$ in 2012 , the percentage of institutions screening outpatients only has increased to $57 \%$ from $50 \%$ in 2012 , and the percentage of those screening select groups of patients only has declined, from $33 \%$ in 2005 to $30 \%$ in 2012 to $17 \%$ in 2018.

Compared with $79 \%$ of institutions in 2012 that reported rescreening patients for distress, $70 \%$ of the respondents to the current survey reported rescreening. This decrease may reflect the greater number in absolute terms of NCCN MIs responding to the current survey and/or the shift of resources from repeat screening to following up on initial screening with triage and referral to appropriate psychosocial resources. Consistent with previous

\section{Table 4. Staffing and Funding for Psychosocial Services ${ }^{a}$}

\begin{tabular}{|c|c|c|c|c|c|}
\hline Staff & $\begin{array}{l}\text { Institutions With } \\
\geq 1 \text { Staff Member }\end{array}$ & \multicolumn{4}{|c|}{ Funding } \\
\hline Neuropsychologists & 8 & 8 & 0 & 5 & 0 \\
\hline Social workers & 17 & 17 & 0 & 2 & 0 \\
\hline Psychiatrists & 11 & 10 & 3 & 9 & 0 \\
\hline Nurses & 13 & 13 & 1 & 2 & 2 \\
\hline Chaplains & 14 & 14 & 3 & 0 & 0 \\
\hline Administrative support & 18 & 18 & 0 & 0 & 1 \\
\hline
\end{tabular}

aTable entries indicate number of institutions out of 18 that provided complete data regarding staffing and funding sources. For example, 15 of 18 institutions indicated they employ at least 1 psychologist; 13 of these institutions subsidize the positions using institutional funds, 3 use grant funds, and 8 use fee-for-service reimbursement. (Note: More than one source may be used.)

bIncludes a patient resource coordinator, cancer information resource nurse, art and music therapists, medical family therapists, and trainees 
results, the intervals and frequencies between the initial screen and rescreening varied widely. Although there may not yet be a standardized approach to rescreening, the details seemed more meaningful than previously, with more than one institution indicating that rescreening is triggered by meaningful clinical events or that distressed patients are rescreened until they score below an established threshold for distress.

Use of brief screening tools seems to have gained greater acceptability, with all respondents using a selfreport measure to screen for distress. In 2005 and 2012 , the percentage of institutions using self-report measures was $63 \%$ and $86 \%$, respectively. The singleitem Distress Thermometer remains the most commonly used self-report screening tool. Recently, Deshields et al ${ }^{10}$ recommended a more global, comprehensive assessment considering multiple components of distress, including physical symptoms. They suggested use of the combined Distress Thermometer and Problem List or the Edmonton Symptom Assessment Scale (ESAS) to better account for physical sources of distress. Use of the Distress Thermometer appears to have remained constant over time. Interestingly, no respondents indicated use of the ESAS for distress screening, although its large-scale use in the clinic-based collection of patient-reported outcomes in a manner similar to distress screening has been documented by at least one MI. ${ }^{11,12}$ The relatively widespread use of the Patient Health Questionnaire and Generalized Anxiety Disorder Assessment scales among respondents seems indicative of the persistent focus on psychological indicators of distress. Their use may also reflect cancer centers' efforts to adhere to ASCO's recommendations for the screening, assessment, and treatment of adult patients with cancer who have symptoms of depression and anxiety. ${ }^{13}$

Consistent with previous results, there is increasing reliance on electronic devices and automation, not only for screening but also to triage patients, facilitate referrals, and document the provision of psychosocial services. For example, although $75 \%$ of respondents in 2012 administered the self-report measure via paper and pencil, only $52 \%$ of institutions did so in 2018 . Similarly, although 14\% of institutions in 2012 triaged distressed patients using automated processes to generate a referral, 33\% of institutions in 2018 triage patients in this manner.

Previous surveys did not inquire specifically about personnel involved in screening and triaging of patients; the current survey appears to be the first to document the role of nurses and medical assistants in these processes. This particular finding highlights the probable need for medical assistants to receive additional training and education on the significance of distress screening as well as the psychosocial aspects of cancer. Social workers continue to play a prominent role in the provision of psychosocial services, while, consistent with the multifaceted nature of distress, psychologists, psychiatrists, and chaplains frequently provide supportive services. Given that the current survey did not inquire about responsibilities specific to each provider, the role of navigators in the provision of psychosocial services remains to be elucidated.

Limitations of the survey should be noted. Among the 27 NCCN MIs surveyed, 4 did not respond, and 1 of these institutions has not participated in any of the 3 surveys conducted to date. Despite an average rate of participation of $86 \%$ across the 3 surveys, findings are only indicative of the subset of institutions that responded to the survey. Similarly, NCCN membership has increased over time, from 18 members at the time of the initial survey, to 27 in June 2018, with the addition of a new member since then. These changes in membership make direct comparisons over time challenging; for some institutions, this was their first opportunity to participate. Finally, as Zebrack et $\mathrm{al}^{14,15}$ demonstrated, the extent to which cancer centers adhere to their own protocols for distress screening is variable. Thus, whether the responses of the panel members who responded to the current survey reflect actual screening practices at their institutions is not known.

\section{Conclusions}

Our findings reflect progress in efforts to adopt and implement the NCCN Guidelines for Distress Management among MIs, particularly with respect to screening, triage, and disposition of distressed patients. Most respondents report the existence of a formal distress screening protocol, with routine tracking of clinical contacts, referrals, and rates of protocol adherence. There is increasing reliance on technology and automation for all aspects of these processes. To date, clinical experience and the ACS $\mathrm{CoC}$ accreditation standard for cancer centers appear to have resulted in greater adoption and implementation of the guidelines. Considerable opportunities for improvement remain, however, reflected in the findings that (1) only about a quarter of those surveyed are currently screening all patients for distress; (2) the specific self-report measures being used are still variable; (3) there is a lack of standardization with respect to when patients are screened and rescreened; (4) there is considerable variability across institutions regarding the services, resources, and staff devoted to treating distressed patients; and (5) data are limited regarding the effectiveness of existing screening protocols. Highlighting the work 
still to be performed, recent publications have called for research to develop an evidence base of effective distress screening and management processes that can be adopted and implemented in clinical and community settings. ${ }^{16,17}$ The newest update of the NCCN Guidelines for Distress Management includes recommendations for implementing the guidelines. Despite progress, there is still much to be determined about the effect of distress screening protocols on patients, providers, and the healthcare system.

Submitted June 7, 2019; accepted for publication September 5, 2019

The authors have no financial interest, arrangement, or affiliation with the manufacturers of any products discussed in the article or their competitors. Drs. Donovan, Corbett, and Riba have disclosed they are panel members of the NCCN Guidelines for Distress Management.

Correspondence: Kristine A. Donovan, PhD, MBA, Moffitt Cancer Center, MRCSCM, 12902 Magnolia Drive, Tampa, FL 33612. E-mail: kristine.donovan@moffitt.org

\section{References}

1. National Comprehensive Cancer Network. NCCN practice guidelines for the management of psychosocial distress. Oncology (Williston Park) 1999; 13:113-147.

2. Cancer Care for the Whole Person: Meeting Psychosocial Health Needs Institute of Medicine. Washington, DC: National Academies Press; 2008

3. American Society of Clinical Oncology. QOPI: The Quality Oncology Practice Initiative. Available at: https://practice.asco.org/qualityimprovement-program/qopi-certification-program. Accessed May 31, 2019

4. Pirl WF, Fann JR, Greer JA, et al. Recommendations for the implementation of distress screening programs in cancer centers. Cancer 2014;120:2946-2954.

5. American College of Surgeons Commission on Cancer. Cancer Program Standards, 2012: Ensuring Patient-Centered Care, Version 1.1. Chicago, IL: American College of Surgeons; 2012.

6. Jacobsen PB, Ransom S. Implementation of NCCN Distress Management Guidelines by member institutions. J Natl Compr Canc Netw 2007;5:99-103.

7. Donovan KA, Jacobsen PB. Progress in the implementation of NCCN Guidelines for Distress Management by member institutions. J Natl Compr Canc Netw 2013;11:223-226.

8. American College of Surgeons Commission on Cancer. Cancer program standards: ensuring patient-centered care, 2016 edition. Available at: https://www.facs.org/quality-programs/cancer/coc/standards. Accessed May 31, 2019

9. Deshields T, Kracen A, Nanna S, et al. Psychosocial staffing at National Comprehensive Cancer Network member institutions: data from leading cancer centers. Psychooncology 2015;25:164-169.

10. Deshields TL, Howrey HL, Vanderlan JR. Distress in oncology: not just a psychosocial phenomenon. J Oncol Pract 2018;14:699-700.
11. Johnstone PA, Bulls HW, Zhou JM, et al. Congruence of multiple patientrelated outcomes within a single day. Support Care Cancer 2019;27: 867-872.

12. Johnstone PA, Lee J, Zhou JM, et al. A modified Edmonton Symptom Assessment Scale for symptom clusters in radiation oncology patients. Cancer Med 2017;6:2034-2041.

13. Andersen BL, DeRubeis RJ, Berman BS, et al. Screening, assessment, and care of anxiety and depressive symptoms in adults with cancer: an American Society of Clinical Oncology's guideline adaptation. J Clin Oncol 2014;32:1605-1619.

14. Zebrack B, Kayser K, Sundstrom L, et al. Psychosocial distress screening implementation in cancer care: an analysis of adherence, responsiveness, and acceptability. J Clin Oncol 2015;33:1165-1170.

15. Zebrack B, Kayser K, Bybee D, et al. A practice-based evaluation of distress screening protocol adherence and medical service utilization. J Natl Compr Canc Netw 2017;15:903-912.

16. Ehlers SL, Davis K, Bluethman SM, et al. Screening for psychosocial distress among patients with cancer: implications for clinical practice, healthcare policy, and dissemination to enhance cancer survivorship. Transl Behav Med 2019;9:282-291.

17. Jacobsen PB, Norton WE. The role of implementation science in improving distress assessment and management in oncology: a commentary on "Screening for psychosocial distress among patients with cancer: implications for clinical practice, healthcare policy, and dissemination to enhance cancer survivorship. Transl Behav Med 2019:9:292-295. 